\title{
A method for the analysis of complex avian song repertoires
}

\author{
DAVID S. CARTER \\ University of California, Riverside, California 92521
}

\begin{abstract}
A new method for the analysis of complex avian song repertoires is introduced. The method consists of sending a tape-recorded bird-song signal through five narrow band-pass filters and then to a five-channel high-speed pen recorder. A coding method that allows a researcher to convert the output from the pen recorder to numbers for later analysis by computer is described.
\end{abstract}

The Kay sonograph has been the standard device for depicting bird vocalizations since the late 1950s. The unit allows a researcher to picture a sound with time on the horizontal axis and frequency on the vertical. Intensity is represented by the darkness of the record. Recent modifications have improved and speeded operation of the sonograph but have not changed the basic function of the device. Attempts to change the strategy of depicting sound on the printed page, such as the zero-crossing of a signal (Greenewalt, 1968; Staddon, McGeorge, Lee, Bruce, \& Klein, Note 1), are not yet common.

There are two disadvantages to using a sonograph, time and data analysis. It takes about $80 \mathrm{sec}$ of printout time and about $45 \mathrm{sec}$ for set-up to make a $2.4-\mathrm{sec}$ section of song. This is not much of a drawback when the repertoire consists of a small number of discrete songs. But with birds possessing large repertoires, adequately sampling a single bird's repertoire is timeconsuming. The second problem is analysis: Most comparisons of interest in bird-song research involve some sort of comparison between the repertoires of different birds. The number of comparisons necessary for two different repertoires increases geometrically as a function of the repertoire sizes. When bird repertoires range across 100 different songs, the labor involved in analyzing repertoire similarities with a decent sample size of birds becomes prohibitive.

The system described here provides a real-time output that can be nominally scaled for later analysis by computer.

\section{FREQUENCY ANALYZING DEVICE}

The frequency analyzing device (FAD) works by receiving a signal from a tape recorder at half-normal speed and sending it simultaneously to five different narrow band-pass filters. Outputs from the filters are rectified and sent to a Beckman Type $R$ dynograph with five Type 9806 ac couplers. Eighteen-inch paper is used at a speed of $25 \mathrm{~mm} / \mathrm{sec}$. Resistors and capacitors smooth out the output signal and balance the channels so that all respond similarly to equal input intensities (see Figure 1).

The center filter frequencies in the FAD are 1, 1.5, 2, 2.5 , and $3 \mathrm{kHz}$. Because the tape is input at half speed, frequencies responded to are twice these figures. The reason for inputting the tape at half speed is to allow the dynograph pens sufficient time to respond and recover. The filters are from KR Electronics in Avenel, New

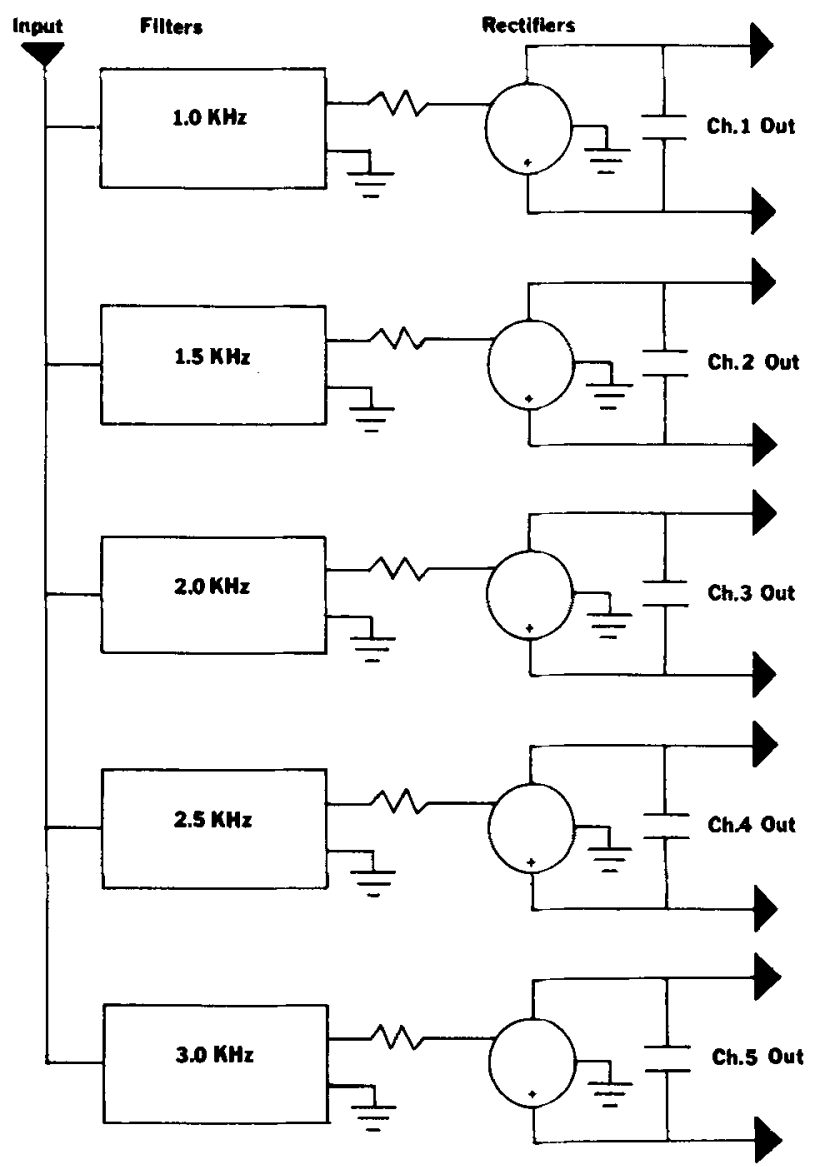

Figure 1. Schematic diagram of FAD. Input is from a taperecorder speaker outlet. Rectifiers produce a more legible output on the pen recorder. 

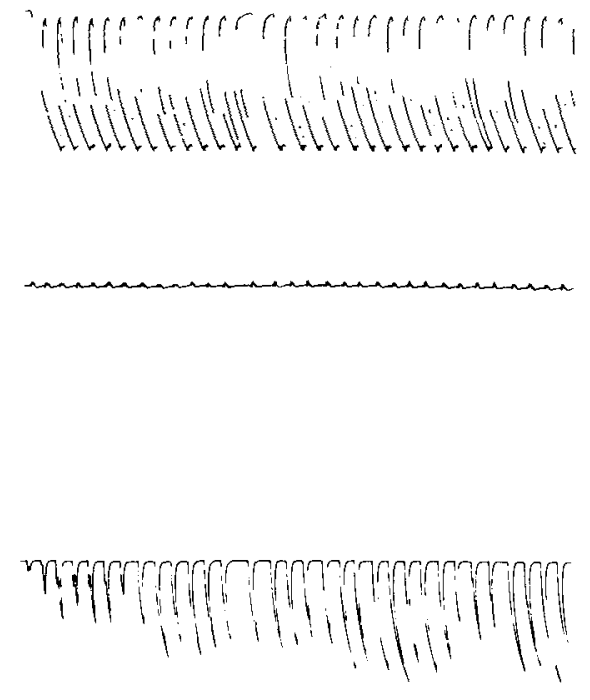

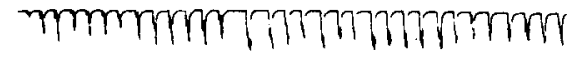

Figure 2. A sample FAD output for the mockingbird SP shown in Figure 4. The 2.kHz channel is on top. Other channels are $3,4,5$, and $6 \mathrm{kHz}$.

Jersey (Parts 1532-1536). Output from the filters attenuates at least $50 \%$ within $10 \%$ of the center frequencies.

Examples of the FAD output for two mockingbird syllable patterns (SP) are shown in Figures 2 and 3. Sonograms of these examples are shown in Figures 4 and 5 for comparison.

\section{FAD CODING}

In developing a nominal scale code for the FAD output, the maximum amount of information is squeezed into a code of limited length. Nine digits is the code length because nine is the largest number that can fit into an INTEGER *4 variable in FORTRAN.

Inspection of Figures 2 and 3 shows that there are a large number of characteristics of FAD output that can be coded to provide various types of information. For example, which channels are responding? What is the shape of the response in a given channel? Is Channel $X$ louder than Channel $Y$ ? Does Channel $X$ respond longer than Channel Y? Which channel onsets first? When the first codes were tried, a given code discriminated readily between most SPs but it confused others. When the code
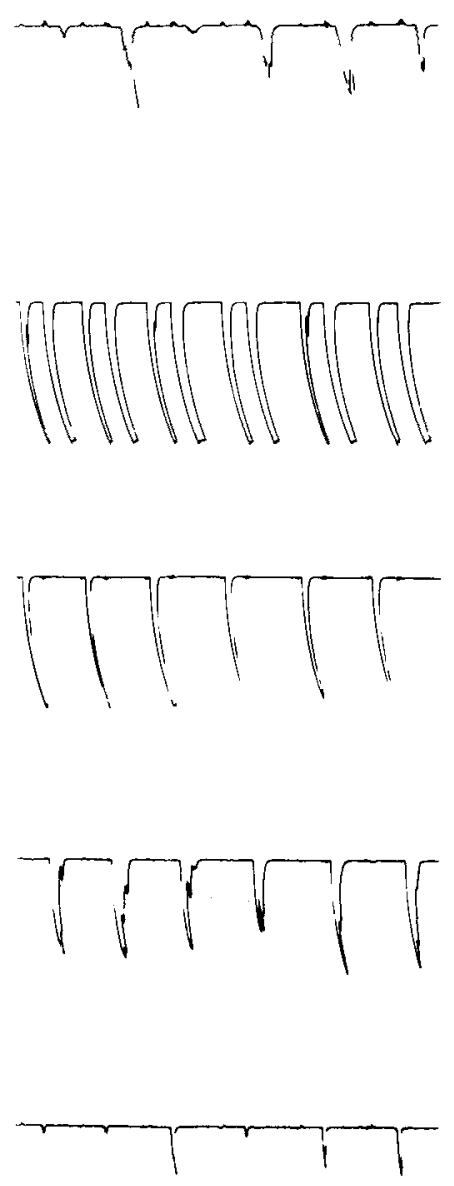

Figure 3. A sample FAD output for the mockingbird SP shown in Figure 5. The $2 \cdot \mathrm{kHz}$ channel is on top. Other channels are $3,4,5$, and $6 \mathrm{kHz}$.

was rewritten, the new code inevitably confused other sets of SPs. No single fixed code was adequate. As a consequence, a branching code was developed.

"Branching code" refers to the fact that the first digit identifies the SP as one of eight general types. These are predicated on whether the shape of the pen response is coded the same in all channels, whether the SP consists of a repeated-note motif, and whether the $2-\mathrm{kHz}$ channel is responding. The remaining eight digits always code the same set of characteristics within each of the eight general types. A general description of the characteristics coded follows.

\section{TYPE}

This two-digit code describes the shape of the pen sweep in a particular channel. Of the 99 possibilities, 85 were used in coding the songs of 18 mockingbirds. The codes for all single- and double-syllable SPs were established a priori, and more complex patterns were assigned a code as they were encountered.

\section{ALT ONSET and ALT LOUD}

These codes are used when one of the 3-, 4-, and $5-\mathrm{kHz}$ channels responds and the $2 \cdot \mathrm{kHz}$ channel 


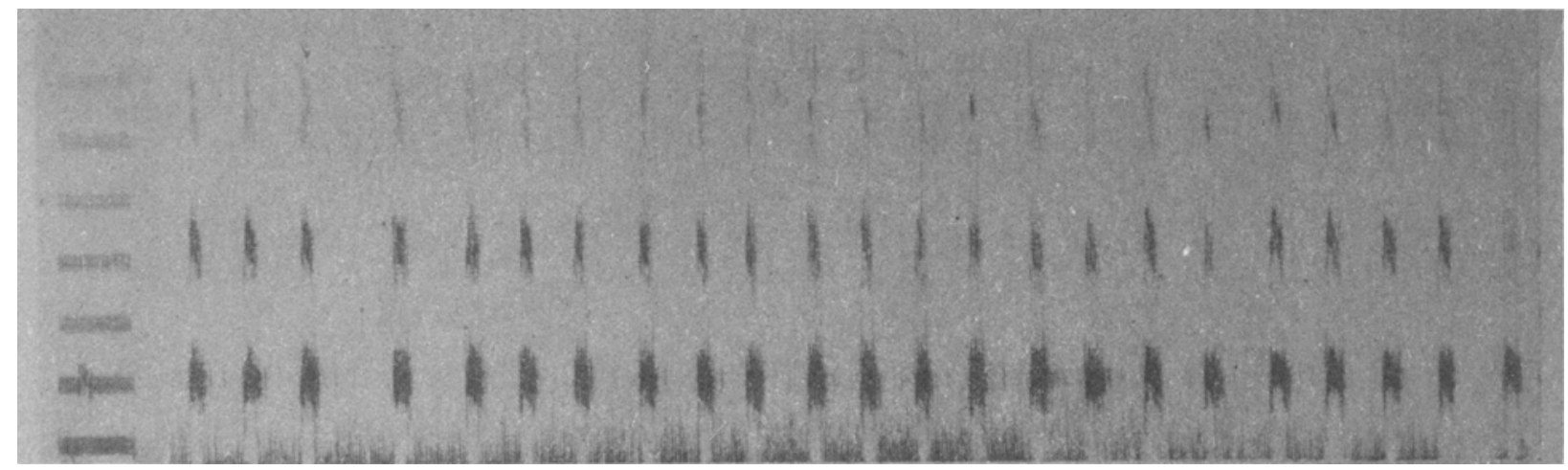

Figure 4. Sonogram of the syllable pattern shown in Figure 2. Frequency markers are at every $1 \mathrm{kHz}$.

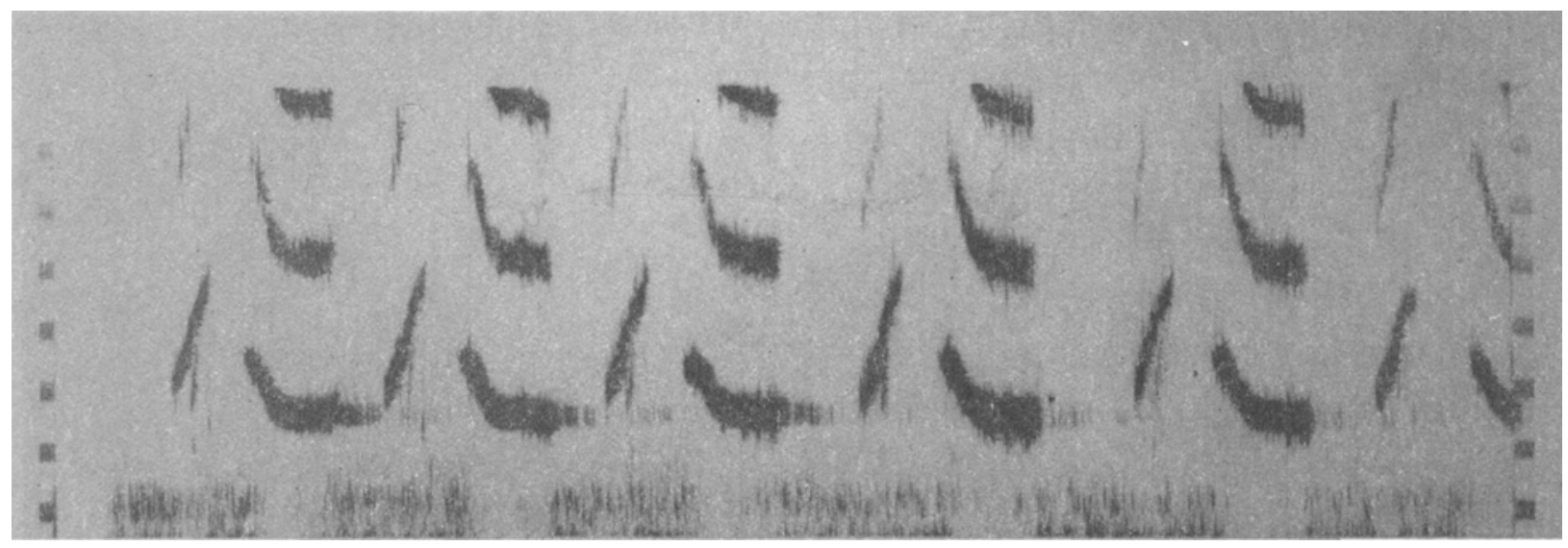

Figure 5. Sonogram of the syllable pattern shown in Figure 3. Frequency markers are at every $1 \mathrm{kHz}$.

responds. It compares the $2-\mathrm{kHz}$ with the other channel in regard to onset time and loudness. It also specifies which of the $3-, 4-$, and $5-\mathrm{kHz}$ channels is responding. The minimum difference for specifying that one channel precedes another is $2 \mathrm{~mm}$. One channel is defined as louder than another when all peaks in one channel are higher than the peaks in the other or when the median peak in one channel is at least twice as high as the median peak in the other.

\section{STD ONSET, STD LOUD, and STD LENGTH}

These three codes compare two of the 3-, 4-, and $5 \cdot \mathrm{kHz}$ channels with regard to onset, intensity, and dwell. They are used whenever only two of the three channels respond or when all three respond but no previous number in the code specifies that all three are responding. When this code is used and all threc channels are responding, only the first two channels are considered.

\section{ODD ONSET, ODD LOUD, and ODD LENGTH}

These codes are used whenever a previous code specifies that the 3-, 4-, and $5-\mathrm{kHz}$ channels are all responding. There is too much information to code in one digit if all of the ordinal possibilities among three channels are coded, so a code was developed to specify the channel that differs most from the other two on a particular measure.

\section{CHANNEL}

This code is used to indicate which of the 3-, 4-, and $5-\mathrm{kHz}$ channels are responding if that information has not already been coded.

\section{SPACE}

This code indicates the space normally found between the SPs relative to the length of the particular SP.

\section{SYMMETRY}

This code indicates whether the SP is symmetrical or misbalaned to the right or left. When there is some doubt regarding the direction to code a misbalanced SP, precedence is given to the channel with the highes frequency. This code and the SPACE code are not used 
unless there is little other codable info in.tion available for the SP. This is because of the ob rivius possibilities for ambiguity in coding these measures

\section{EXAMPLES}

The final code for the output showr in Figure 2 is 130102040. The general type code value of 1 in the first position indicates that the activated channels are not responding with the same TYPE code, the SP is not made up of a repeated pattern, and the $2 \cdot \mathrm{kHz}$ channel is responding. The 3 in the second position indicates that, of the 3-, 4-, and $5-\mathrm{kHz}$ channels, only the $4-\mathrm{kHz}$ channel is responding. The 01 in the next two positions defines the TYPE, a simple peak, in the channel that is responding. The 02 in the following two positions designates the shape of the response in the $2-\mathrm{kHz}$ channel. The next 0 means that the two responding channels start simultaneously. The 4 indicates that the $2-\mathrm{kHz}$ channel has a louder response than the $4 \cdot \mathrm{kHz}$ channel. The last position in this example is not used; 0 is inserted as a filler.

The code for the output shown in Figure 3 is 229131010. The general type code value of 2 designates that there are at least two TYPE codes among the responding channels, it is not a repeated-pattern type of $\mathrm{SP}$, and the $2 \mathrm{kHz}$ channel is not responding. The salient two of the responding channels are clearly the 3- and $4-\mathrm{kHz}$ channels. The TYPE code of the first of these is 29 and designates a peak followed by a broad response. The TYPE code for the second of the salient two channels is 13 and designates a noise-peak-noise response. The following 1 indicates that the $3-\mathrm{kHz}$ channel precedes the $4 \cdot \mathrm{kHz}$ channel. The next 0 means that there is no intensity difference between the two channels. The next 1 indicates that the $3-\mathrm{kHz}$ channel is longer than the $4-\mathrm{kHz}$ channel. As in the last example, the trailing 0 is a space filler.

\section{DISCUSSION}

FAD and the coding system have proven useful for analyzing the songs of the mockingbird. Regional differences in song types can readily be discerned along with regional differences in some of the song parameters. In addition, the method allows sequential analyses for any number of song parameters. These sequential analyses show the extent to which the characteristics of one SP are correlated with those of the subsequent SP. Such analyses are difficult without the use of a computer.

FAD could be improved by feeding the output from the filters directly to an on-line computer for the timeconsuming coding chore. This is most feasible if the subjects are observed in an environment with no background noise. Unfortunately, typical in-habitat bird recordings have high background noise levels and signals of various intensities. These most often require a great deal of editing.

\section{REFERENCE NOTE}

1. Staddon, J. E. R., McGeorge, Lee W., Bruce, R. A., \& Klein, F. F. A simple method for the rapid analysis of animal sounds. Unpublished manuscript, 1979.

\section{REFERENCES}

Greenewalt, C. H. Bird song: Acoustics and physiology. Washington, D.C: Smithsonian Press, 1968.

VERNER, J. Complex song repertoire of male long-billed marsh wrens in eastern Washington. Living Bird, 1975, 14, 263-300.

\footnotetext{
(Received for publication August 20, 1979;
} revision accepted December 14,1979 .) 